\title{
An Improved Escherichia Coli Bacterium Detection in Microchannel Based on Dielectrophoresis Impedance Measurements
}

\author{
Alireza Ebrahimi a,b, Morteza Fathipour ${ }^{\text {b }}$, Maryam Fathollahzadeh ${ }^{\text {b,c,* }}$ (D) \\ a School of Electrical Engineering, Islamic Azad University, Tehran, Iran \\ ${ }^{b}$ School of Electrical and Computer Engineering, University of Tehran, Tehran, Iran \\ ${ }^{c}$ Chemistry and Processing Group, Chemistry and Materials Division, Niroo research Institute, Tehran, Iran
}

\section{ARTICLE INFO}

Received: 26 May 2019

Revised: 22 July 2019

Accepted: 19 August 2019

Available online: 21 August 2019

\section{K E Y W O R D S}

Clausius-Mossotti Factor DEPIM

Escherichia Coli

Microfluidics

\section{A B S T R A C T}

In this study, a bacterial detection technique utilizing benefits of both positive and negative dielectrophoresis has been simulated with COMSOL Multiphysics. The two dimensional proposed model has six microelectrodes across microfluidic channel. Four of them were positioned on the upper side and the rest were positioned on the bottom side of the microchannel, which serve as a bacteria concentrator using negative dielectrophoresis and as a bacteria detector using positive dielectrophoresis, respectively. In this simulation, the target particle is Escherichia coli that was flowing into the microchannel and repelled under negative dielectrophoretic force exerted by the upper side microelectrodes, and were pushed toward the bottom side microelectrodes situated at the downstream. Finally, concentrated bacteria have been captured and detected by dielectrophoretic impedance measurement method. The numerical simulation proved that negative dielectrophoretic force could increase sensitivity with respect to the absence of the negative dielectrophoretic force and eventually, results represented that 80 percent of releasing E. coli bacteria trapped near bottom side microelectrodes. 


\section{G RA P H I C A L A B S T RACT}

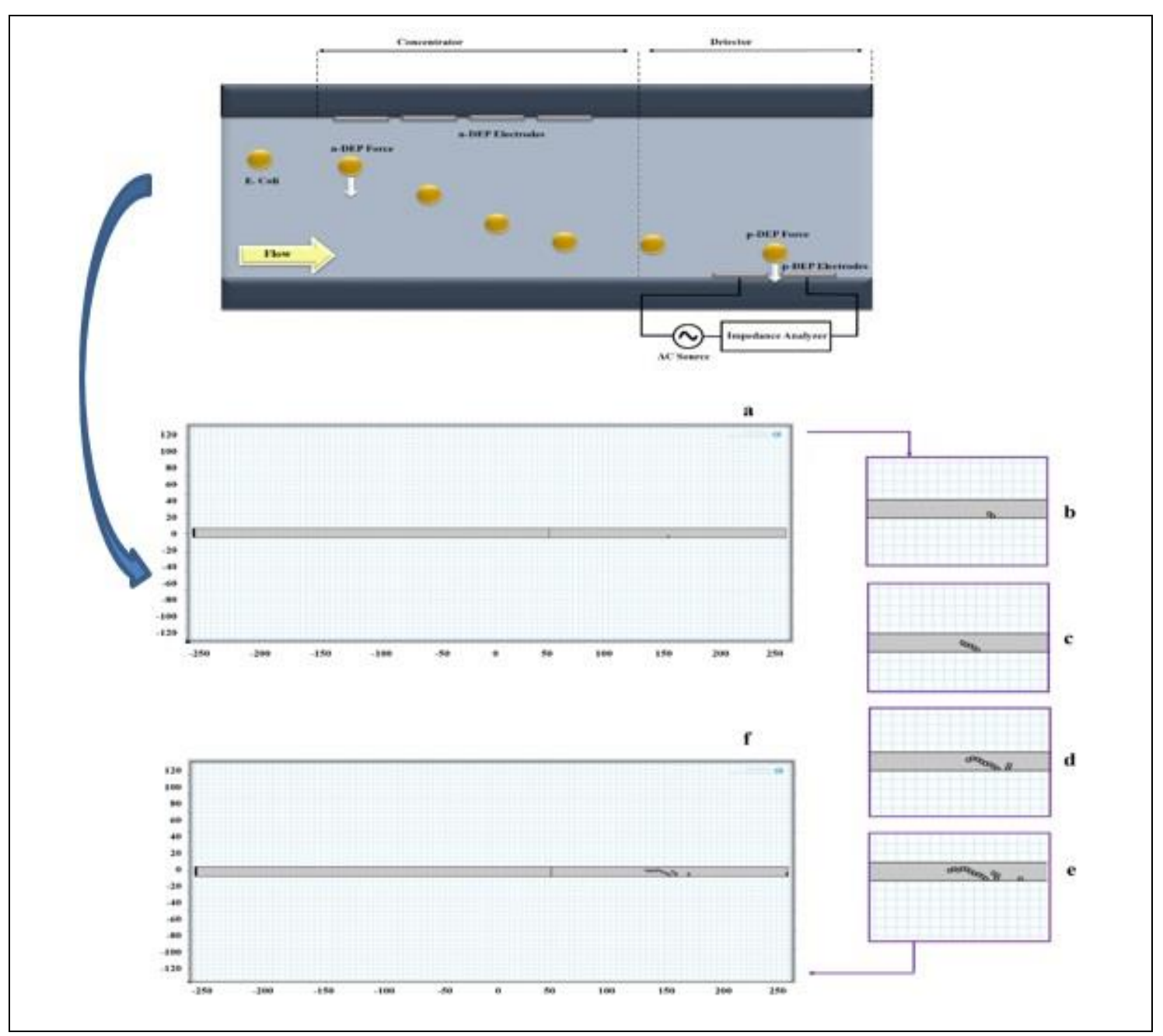

\section{Introduction}

Dielectrophoresis (DEP) was first introduced by Pohl [1,2] to describe the motion of particles due to dielectric polarization effects in non-uniform applied electric fields. DEP is an efficient, electrokinetic effect with huge application for manipulating sub-micron species. In fact, DEP represents a force which is exerting on a dielectric particle when it is subjecting to a non-uniform applied electric field [3-8]. It does not mean that the dielectric particles need to be charged, so that all particles show DEP reaction in the attendance of electric fields. In addition, the strength of the DEP force fully depends on the electrical properties of suspended medium and particles, frequency of the electric field and shape and size of particles. Therefore, DEP occurs in AC and DC electric fields [9-14].

DEP has been proven as an adaptable mechanism to transportation, accumulation, separation and characterization of submicron bio-particles in microfluidic systems. The integration of DEP systems into the microfluidics provides the inexpensive, fast, highly sensitive, selective and label-free detection of bio-species [15-23].

Microfluidics emerged in the beginning of the 1980s and deals with the manipulation of fluids that are geometrically constrained to a small, usually sub-millimeter systems. It is an innovative field at the intersection of engineering, physics, biochemistry, nanotechnology, and biotechnology, with 
practical applications in the design of low volume systems of fluid. Microfluidics is used in the development of inkjet printers, DNA chips, micro-propulsion, and micro-thermal technologies [24-31].

One of the most interesting fields in microfluidics is particle investigation and many researchers have focused on cell manipulation techniques for biomedical and commercial applications, including nanoparticle separation, concentration, identification, trapping and transporting [32]. DEP is a desirable candidate for manipulation, separation and concentration of a wide range of particles, ranging from macromolecules to every types of bacteria, viruses and parasites [8, 33-39]. It means that, researchers can utilize these benefits to investigate the various important and especially vital particles. One of the common DEP methods is dielectrophoretic impedance measurement (DEPIM) that can measure impedance changes of the particle in microchannel in the presence of non-uniform applied electric fields. Thus, it is possible to predict and control the movements of particles in microchannel [40-43].

Undoubtedly, the evaluation of the level of bacterial contamination in a production process or in the final products is one of the major challenges of the food industry, as a prevalence of harmful bacteria in final products may cause human health threat. Therefore, a large variety of structures, ranging from simple planar geometries to fully complex designs, is now fabricated or under investigation to insure the product's health [44-51].

E. coli is a gram-negative, rod-shaped, coliform bacterium of the genus Escherichia that is commonly found in the warm-blooded organisms. Some E. coli strains are harmless, but the other types cause serious food poisoning, and are widely resulted in some kind of final food products recalls because of food contaminations.
Many researchers investigated simulation of submicron particle's behavior and their activity. Simulating procedures before starting any fabrications have various advantages like lowering the price of fabrication by studying on the procedures details or avoiding unnecessary contact with the harmful particles like pathogenic bacteria or perilous viruses prior to studying their behavior during the measurement processes. In simple term, researchers use simulation for achieving experience in a low-cost, low-risk environment. In the present work, the computational software COMSOL Multiphysics was applied to simulate a lab on a chip (LOC) device for detection of bacteria utilizing DEPIM. COMSOL Multiphysics is a software for simulating every electrical, mechanical and chemical systems.

\section{Experimental}

\section{Dielectrophoretic Force}

In order to simulate a LOC device for detection of microparticles such as bacteria, it is needed to define all forces that exerted to the target particles in designed microchannel and in presence of non-uniform electric fields. The dielectrophoretic force exerted on a simple spherical particle of radius $\mathrm{R}$ is defined by equation (1) as follows [5],

$F_{D E P}=2 \pi R^{3} \varepsilon_{m} f_{C M} \mathbb{V E}^{2}$

Where $\varepsilon_{\mathrm{m}}$ is the permittivity of the suspending medium, $\nabla E^{2}$ is the gradient of the square of the root-mean-square of the electric field and $\mathrm{f}_{\mathrm{CM}}$ is the Clausius-Mossotti factor, defined as:

$f_{C M}=\operatorname{Re}\left[\left(\varepsilon_{p}^{*}-\varepsilon_{m}^{*}\right) /\left(\varepsilon_{p}^{*}+2 \varepsilon_{m}^{*}\right)\right]$

Where $\varepsilon_{p}^{*}$ and $\quad \varepsilon^{*}{ }_{m}$ are the complex permittivity of the particle and the suspending medium respectively. Complex permittivity of the particle or suspending medium define as follows; 
$\varepsilon^{*}=\varepsilon+\frac{\sigma}{j \omega}$

Where $\sigma$ is the conductivity of the particle $\left(\sigma_{\mathrm{p}}\right)$ or the suspending medium $\left(\sigma_{\mathrm{m}}\right)$ and $\omega$ is angular frequency that is applying to the particle. All polarizable particles has shown a positive or negative dielectrophoresis ( $p$-DEP or $n$-DEP) induced movements depending on the value of the $\operatorname{Re}\left[f_{\mathrm{CM}}\right]$. The $\operatorname{Re}\left[f_{\mathrm{CM}}\right]$ depends on the applied frequency, permittivity and conductivity of the particle and the suspending medium [52]. By changing these parameters, target particle movements can be directed according to its DEP effects. For instance, higher $\varepsilon_{\mathrm{p}}^{*}$ than $\varepsilon_{\mathrm{m}}^{*}$ leads to positive Re $\left[f_{C M}\right]$, hence particles experience $\mathrm{p}$-DEP force and go towards the higher electric field gradient region (edges of microelectrodes). On the other side, when $\varepsilon^{*}$ is higher than $\varepsilon^{*}{ }_{p}$ in the microchannel, Re $\left[f_{C M}\right]$ becomes negative and particles go toward the low electric field gradient region (far from the edges of microelectrodes) [53].

When a direct current (DC) electric field is applied, the frequency dependent component of the Clausius-Mossotti factor approaches zero, thus it could be defined in terms of the conductivities of the particle and the suspending medium.

$f_{C M}=\left(\sigma_{p}-\sigma_{m}\right) /\left(\sigma_{p}+2 \sigma_{m}\right)$

Where $\sigma_{\mathrm{p}}$ the conductivity of the particle and $\sigma \mathrm{m}$ is the conductivity of suspending medium.

\section{Drag Force}

In fluid dynamics, drag (sometimes called fluid resistance or fluid friction) is a force, acting opposite to the relative motion of any particles moving with respect to a surrounding fluid. It can take place between two fluid layers or surfaces or a fluid and a solid surface. Unlike other resistive forces, which are commonly independent of velocity, drag force fully affected by velocity. Drag force is related to the velocity in laminar flow mode and to the square of velocity in a turbulent flow.
Even though the main reason of drag forces refer to viscous friction, the turbulent drag is independent of it [54]. Meanwhile drag force causes decrement of the fluid velocity relative to the solid object in the fluid's direction. For a spherical particle in a fluid of viscosity $\eta$, Stoke's law gives the drag force by equation (5),

$F_{\text {Drag }}=-6 \pi \eta a v$

Where $\eta$ is the dynamic viscosity of the medium and $v$ is the particle velocity that moves in the surrounding medium.

\section{E. coli Structure}

Bacteria are microscopic single-celled organisms that exist in many different sizes and shapes. Bacteria were classified based on their morphology, or shape and appearance. The three basic shapes of bacteria are spherical, rod shaped and spiral. Each morphology-based group has at least some disease-causing representatives [55].

Escherichia coli (E. coli) gram-negative ubiquitous bacteria normally live in the intestines of humans and warm-blooded animals. Most $E$. coli are harmless and actually are an important part of a healthy human intestinal tract. Nevertheless, some strains have evolved the capability to cause both intestinal and extraintestinal illnesses. Some E. coli are pathogenic, it means that they can cause illness. These types of $E$. coli can transmit through contaminated water or food or through contact with animals or humans [56,57].

Every cells of bacteria have complex structure, but most of them consist of three shells, cell membrane, and cell cytoplasm and cell nucleus. As shown in Figure 1, the outer layer is membrane, cytoplasm is the middle one and nucleus is the inner layer [5].

Every shells have specific electrical properties and different radius. Before starting simulation, these three complex shells in real structure converted to a simple circular model (2D simulation) with equivalent conductivity, 
permittivity and radius to use in COMSOL Multiphysics 2D simulation processes. As illustrated in Figure 2, the equivalent radius is the total radius of cell from center point of

(a)

Figure 1. a) Spherical model of $E$. coli bacterium b) 3shell equivalent model

Homogenous sphere model

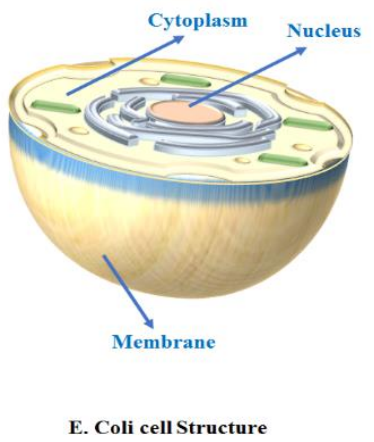

nucleus to the outer wall of membrane or in a simple term, the equivalent radius of cell is the total size of membrane radius, cytoplasm radius and nucleus radius.

(b)

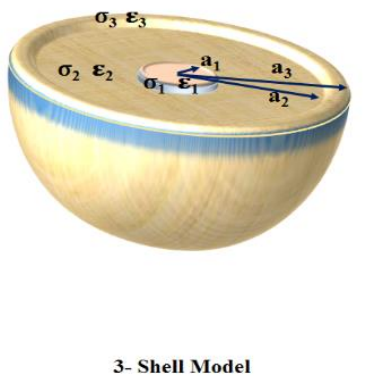

(c)

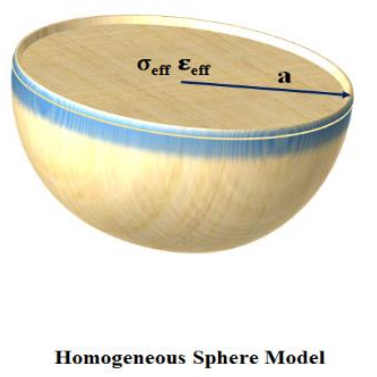

Figure 2. a) Equivalent radius of cell. b) Approximate steps of equivalent complex permittivity of 3shell Sphere
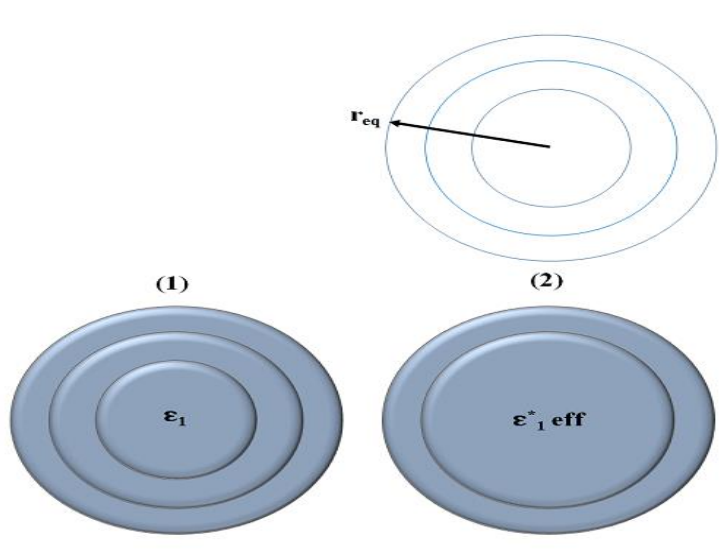

(a)

Computation of equivalent complex conductivity and complex permittivity is extremely different from computing equivalent radius. For instance, we calculate the equivalent permittivity use the equations below [5],

$\varepsilon_{1 e f f}^{*}=\varepsilon_{2}^{*} \frac{\left(\frac{r_{2}}{r_{1}}\right)^{3}+2 \frac{\varepsilon_{1}^{*}-\varepsilon_{2}^{*}}{\varepsilon_{1}^{*}+2 \varepsilon_{2}^{*}}}{\left(\frac{r_{2}}{r_{1}}\right)^{3}-\frac{\varepsilon_{1}^{*}-\varepsilon_{2}^{*}}{\varepsilon_{1}^{*}+2 \varepsilon_{2}^{*}}}$

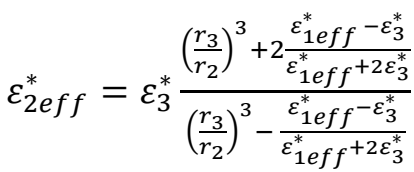
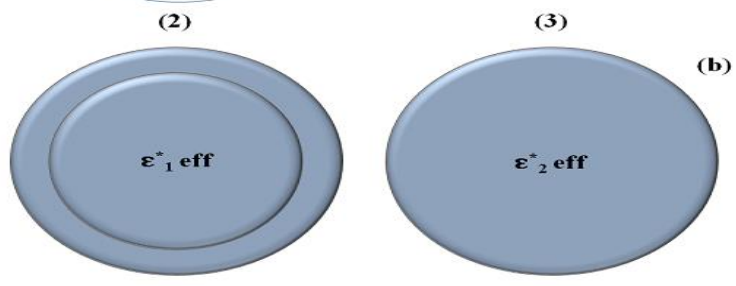

Equations (6) and (7) called 3-shell equations and can be generalized to multi-shell bioparticles. Therefore, multi-shell equations are quite important for particle simulation in every micro-electro-mechanical systems (MEMS).

After achieving the 2D model of $E$. coli and its properties, simulation of proposed LOC device has any required equation in COMSOL Multiphysics.

As mentioned before, COMSOL Multiphysics provides unique workflow for electrical, mechanical and chemical systems. Also, it is a software for finite element analysis and multiphysics simulation. It allows contractual physics-based user interfaces and coupled systems of partial differential equations (PDEs).

\section{Results and discussion}


Proposed model

In this study, the movements of $E$. coli in a designed simple rectangular microchannel have been simulated. As shown in Figure 3, the microchannel height and length are $10 \mu \mathrm{m}$ and $500 \mu \mathrm{m}$ respectively. It should be noted in $2 \mathrm{D}$ simulation, the material and electrical properties of microchannel are not decisive.

Six microelectrodes were used in microchannel that four of them are on the upper side and two of them are on the bottom side (Figure 3). The height and length of microelectrodes are $0.1 \mu \mathrm{m}$ and $50 \mu \mathrm{m}$, respectively and the gap between them is $5 \mu \mathrm{m}$. The gap between the last microelectrode on the upper side of microchannel and the first microelectrode on the bottom side is $150 \mu \mathrm{m}$.

\section{Applied modules and calculations}

In order to estimate electric field distribution, electric field gradient and velocity lines of the target Bacteria, as well as bacteria tracing in the microchannel and DEPIM effects, simulation was carried out based on electrical modules built with COMSOL Multiphysics. Three applied physical modules of COMSOL Multiphysics are: 1) laminar flow, 2) electric current and 3) particle tracing for fluid flow. As known, the type of fluid that is flowing in the microchannel and applied electric field are independent of time (steady state) and clearly the location of target bacteria in the microchannel changes over time and it is completely time dependent. Thus, the steady state analysis was used for laminar flow and electric current modules, while time dependent analysis was used for particle tracing for fluid flow module. Two steps approach was employed for model analyzing, steady state analysis in the first step and time dependent analysis in the second step that apparently depends on the results of the first one.

In this simulation, it is assumed that a fluid flows in the microchannel is water and all six microelectrodes made of chromium. Table 1 shows the parameters and their values used for predicting the model.

At first, equivalent radius was calculated, as well as equivalent complex permittivity and conductivity of E. coli. Complex 3-shells E. coli was converted to simple circular model (for 2D simulation). There are potential approaches ahead, 1) Entering equations and define electrical properties of target bacteria as parameters or 2) calculate equivalent properties by the user. The first way was selected, since it might be needed to evaluate system by changing parameters without calculating in different frequencies as well as different targets or other suspended medium.

As mentioned before, the rectangular microchannel has been drawn. After introducing dimensions of proposed microchannel in geometry section of software, the boundary conditions was determined as well as fluid velocity in each module subsections. Afterward, the type of fluid was specified. In addition, a fluid flow from left side to right side is assumed to be in laminar mode. 
Figure 3. 2D model of the microfluidic channel and the $n$ DEP and p-DEP microelectrodes

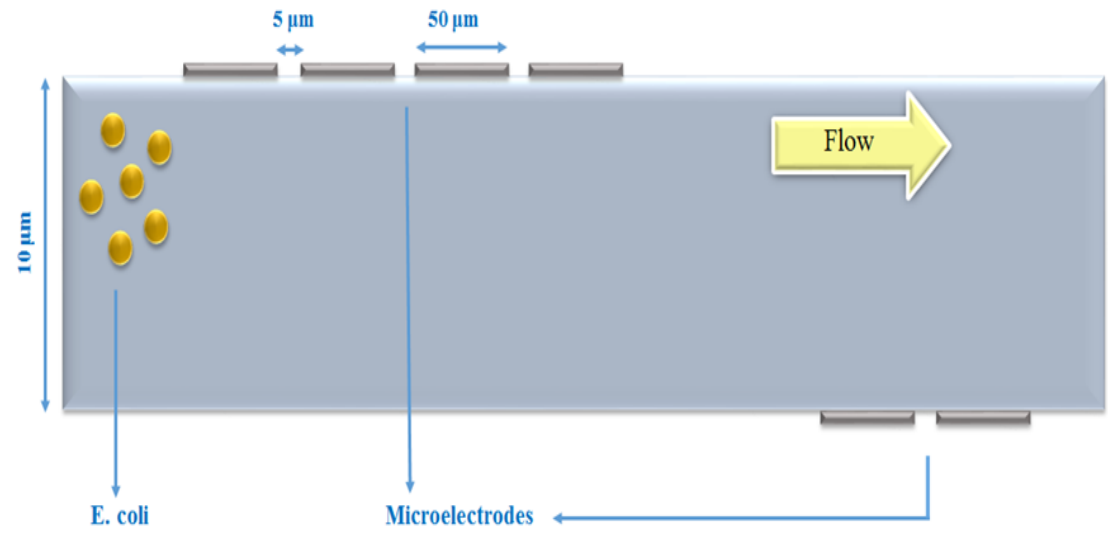

Table 1. The dielectric properties and of $E$. coli [8] and medium used in the simulations

\begin{tabular}{cc} 
Parameter & Value \\
Cell radius (Nucleus + Cytoplasm) & $1 \mu \mathrm{m}$ \\
Cytoplasm conductivity & 60 \\
Cytoplasm relative permittivity & $0.2 \mathrm{~S} / \mathrm{m}$ \\
Membrane radius & $0.08 \mu \mathrm{m}$ \\
Membrane conductivity & $0.25 \mu \mathrm{S} / \mathrm{m}$ \\
Membrane relative permittivity & 6 \\
Medium conductivity & $100 \mu \mathrm{S} / \mathrm{m}$ \\
Medium relative permittivity & 80 \\
Medium thermal conductivity & $0.6 \mathrm{~W} / \mathrm{m} . \mathrm{K}$ \\
Medium dynamic viscosity & $0.89 \mathrm{mPa} . \mathrm{s}$ \\
Medium ratio of specific heat & 1 \\
Chromium relative permittivity & 1 \\
Poisson's ratio & 0.21 \\
Chromium thermal conductivity & $93.7 \mathrm{~W} / \mathrm{m} . \mathrm{K}$ \\
\hline
\end{tabular}

In fluid dynamics, laminar flow takes place when a fluid flows in parallel layers, with no interruption between them. There are two kinds of fluid flow: 1) laminar flow and 2) turbulent flow. The difference between laminar and turbulent flow cleared by the definition of Reynolds Number (Re). This number is an important dimensionless quantity in fluid mechanics used to predict flow patterns in different fluid flow situations. The Reynolds number, defined as:

$R e=\rho V D / \mu$

Where $\rho$ is the density of the fluid, $\mathrm{V}$ is the velocity of the fluid with respect to the object, D is a characteristic linear dimension (travelled length of the fluid) and $\mu$ is the dynamic viscosity of the fluid. Laminar flow takes place at low Reynolds numbers, where viscous forces are dominant, and characterized by smooth fluid motion (laminar flow motion).

Upper and lower edge of the microchannel were defined as a wall and after that, boundary conditions for each walls for solving Maxwell's law has been determined. In order to find velocity field, Continuity equation (9) and Navier-Stocks equation (10) should be solved with the following boundary conditions: (1) noslip at the walls, (2) determined flow velocity at the microchannel inlet and (3) zero pressure in the microchannel outlet.

$\nabla \cdot \overline{\mathrm{u}}=0$ 
Where u define velocity of fluid,

$\rho .(\partial \overline{\mathrm{u}} / \partial \mathrm{t})+\rho \bar{u} . \nabla \overline{\mathrm{u}}=-\nabla \mathrm{P}+\eta \cdot \nabla^{2} \overline{\mathrm{u}}$

Where $\rho, \eta$ and P are density, viscosity and pressure of medium respectively. As illustrated in Figure 4, the velocity of fluid in the middle of simple rectangular microchannel is higher than that of near the walls since drag force exerted on the fluid near walls.

In experimental applications, peristaltic pump injects the fluid into the microchannel with exact desired initial velocity (dependent to their calculations). In this simulation, the

Figure 4. The velocity of the fluid during microchannel

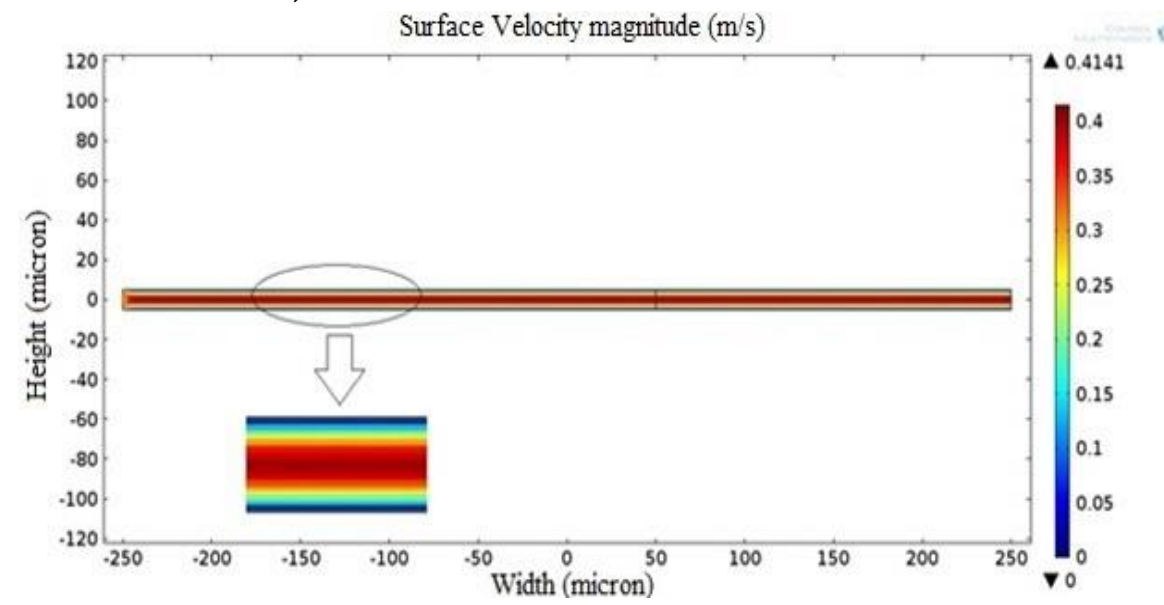

Figure 5. A 2D

model microfluidic channel and the $n$ DEP and p-DEP microelectrode for the numerical simulations velocity of fluid was introduced $27 \mathrm{~cm} / \mathrm{s}$ based on microchannel dimensions and E. coli simple circular model.

As shown in Figure 5, in the second step, the voltages applied to the represented 2D model of proposed rectangular microchannel. It was designed 2D simulation truly represents the system because the microelectrodes were long enough compare to their width. For the electric field calculations, voltages of the adjacent microelectrodes are V and grounded (G), respectively.

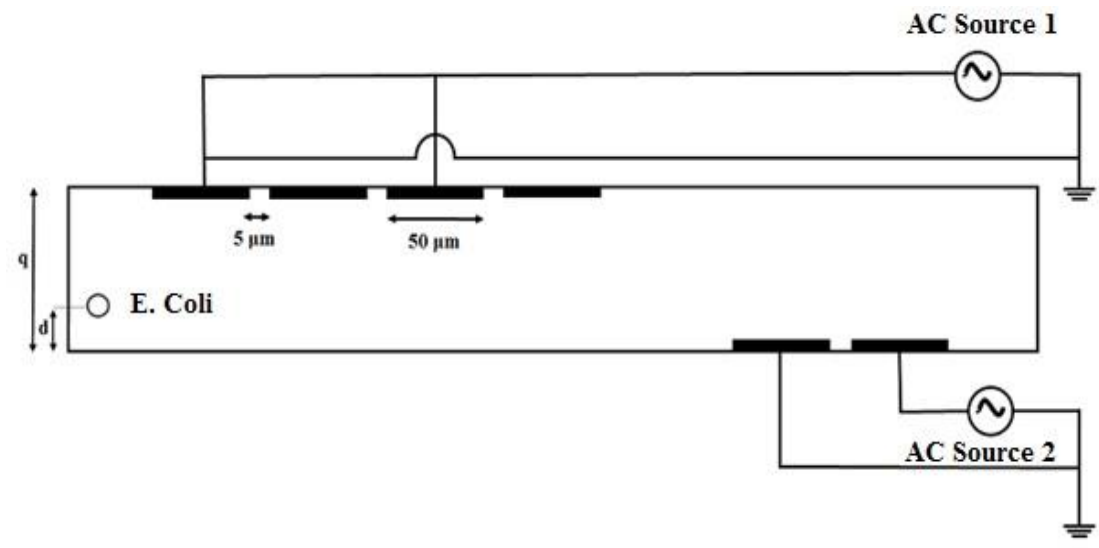

Electric current module of the software with the condition of steady state analysis was used for introducing applied voltage to chromium microelectrodes in the upper and bottom sides of the microchannel. As mentioned before, there are various kinds of limitation for applying voltages to the target bio-particles. Therefore, different applied voltages (V) was tested to ensure damaging of the target $E$. coli was minimized.

In this 2D proposed model the mesh was created automatically by FEM solver and it was used finer mesh near edge of microelectrodes. Figure $6 \mathrm{a}$, shows the variation of the target 
bacteria positions (the initial position of $E$. coli was assumed to be $d=9 \mu \mathrm{m}$ ), after passing three microelectrode gaps (four microelectrodes at the upper side of the microchannel) as a function of applied voltage. Increasing the applied voltage of upper side microelectrodes, leads to change $E$. coli position toward the bottom side of microchannel, but with biology limitations of E. coli, it is better to use the lowest possible voltage. Obviously upper position targets in the microchannel more affected by applied $n$-DEP in the first section of channel than lower position targets, so voltage of $20 \mathrm{~V}$ applied to selected microelectrodes (because of our target limitations). Figure $6 \mathrm{~b}$, shows the electric field distribution in the microchannel based on selected applied voltage.

Before detecting of E. coli, separation of bacteria should be done from other bio-particles in the surrounding medium. This issue has been resolved by a special applied frequency that the behavior of target is in opposite way of other bio-particles in the fluid (maybe it takes several steps because of the similarity behavior of bioparticles against frequency).

In first part of the microchannel (near four microelectrodes on the upper side of the microchannel), dielectrophoretic force exerted on $E$. coli should be negative to push it to the down side of the microchannel as much as possible (due to $E$. coli limitations).

The term crossover frequency is determine for manipulation of every target bio-particles and it specifies the direction of applied DEP. The crossover frequency defined as below [27]: $\omega_{c}=\sqrt{ }\left(\left(\left(\sigma_{m}-\sigma_{p}\right)\left(\sigma_{p}+2 \sigma_{m}\right)\right) / \mid \varepsilon_{p}-\right.$

$\left.\varepsilon_{m} \mid\left(\varepsilon_{p}+2 \varepsilon_{m}\right)\right)$

As shown in equation 11, the crossover frequency depends on the electrical properties of the target bacterium $\left(\varepsilon_{\mathrm{p}}, \sigma_{\mathrm{p}}\right)$ and the surrounding medium $\left(\varepsilon_{\mathrm{m}}, \sigma_{\mathrm{m}}\right)$. It is time to calculate crossover frequency in this study, because all required parameters is define by determining E. coli as target bio-particle and water as surrounding medium. Calculation showed that the crossover frequency of $E$. coli in this proposed model was about $0.5 \mathrm{MHz}$.

In the next step, two major problems existed with COMSOL Multiphysics. First, the lack of understanding of software about 2 different frequencies in one system and second the assumption of moving particles as points. For instance, entrance height of rectangular microchannel is $10 \mu \mathrm{m}$ and in reality, the maximum number of five $E$. coli bacterium (radius of $1 \mu \mathrm{m}$ ) can enter at the same time, however software, software does not consider any limitations of entering bacteria at a same time.

The first problem has been solved by utilizing a simple technique. Two rectangular microchannel has been drawn and connected them from their height to each other and defined the entrance and exit of microchannel as same as designed microchannel. The important point is, the wall between two rectangles is virtual and apparently, it does not need to define boundary conditions. Figure 7, shows two shapes that build designed rectangular microchannel (middle wall is not real wall). 
Figure 6. a) The variation of E. coli position after passing three microelectrodes gaps as a function of applied voltage. b) Electric field distribution in the microchannel based on selected voltage of $20 \mathrm{~V}$

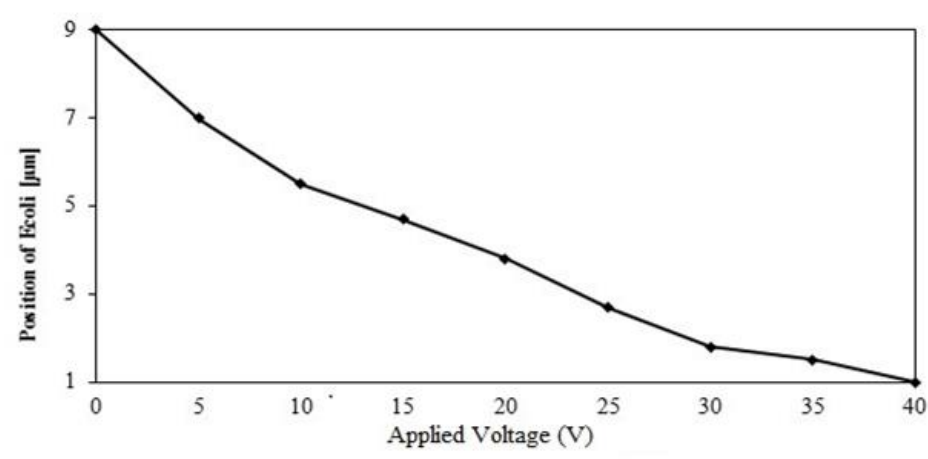

(a)

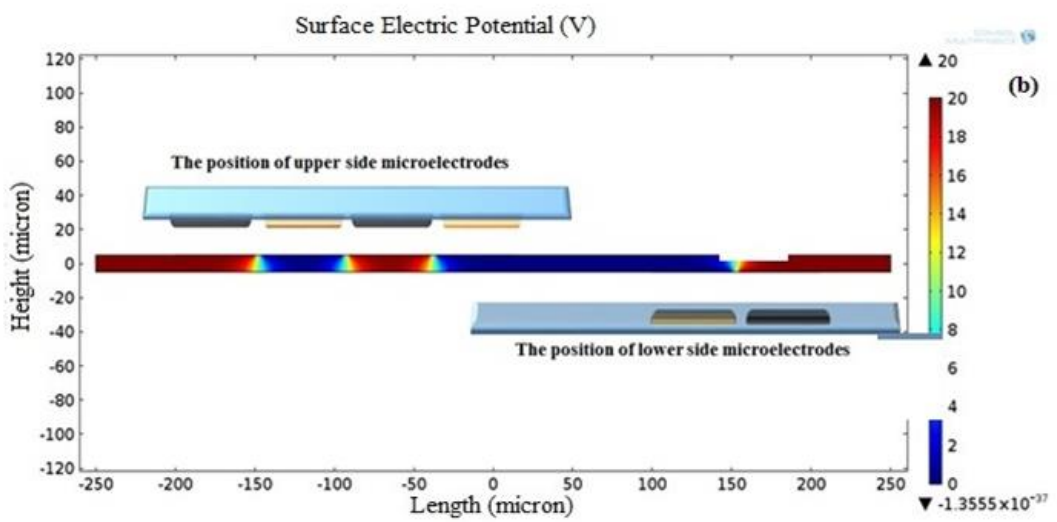

Figure 7. Proposed rectangular microchannel with virtual wall

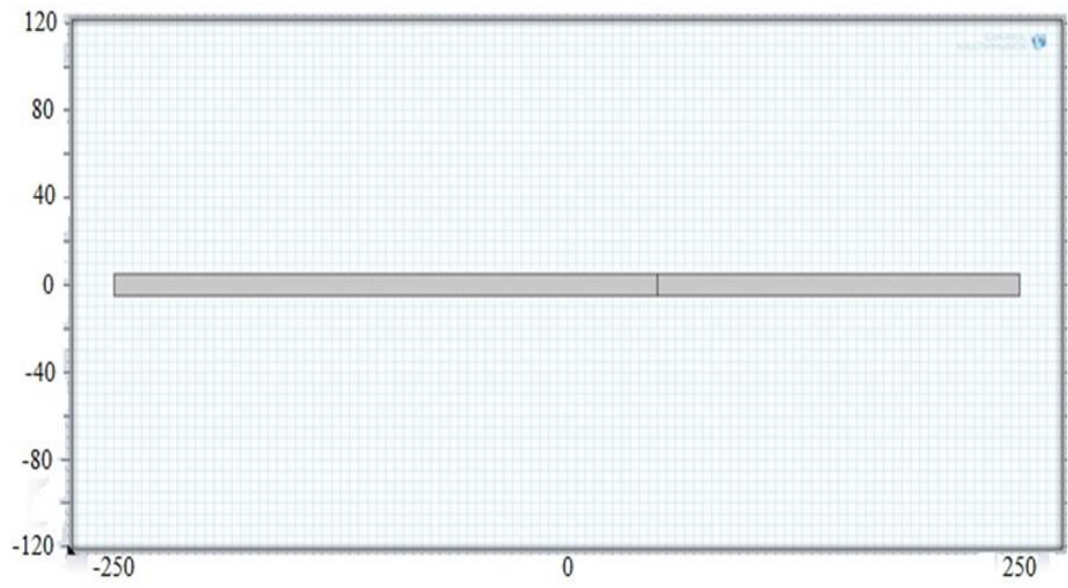

Figure 8. E. coli trajectory based on time dependent analysis of particle tracing for fluid flow module

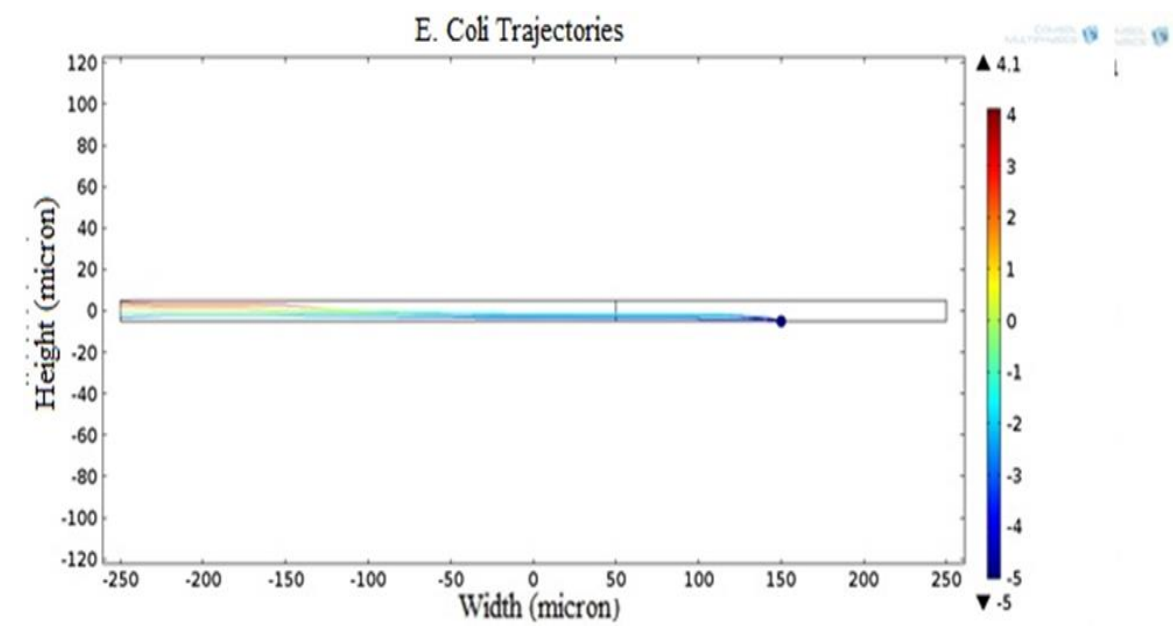


By utilizing this technique, different frequencies could be applied to one system without any frequency interferences. As mentioned before, in the frequency of $0.5 \mathrm{MHz}$, the Calusius-Mossotti factor was zero and consequently dielectrophoretic force exerted on E. coli was zero.

\section{Results}

N-DEP should exerted on the targets to push E. coli bacteria to the bottom side of the microchannel. Afterward, a frequency lower than $0.5 \mathrm{MHz}$ has been selected $(1 \mathrm{KHz})$ for the first four upper side microelectrodes to have nDEP at first section of the microchannel and a higher frequency of $1 \mathrm{MHZ}$ has been chosen for two bottom side microelectrodes to have p-DEP at the second section of microchannel.

Detection of $E$. coli bacteria was the main goal of this study. As represented above, two chromium microelectrodes in the bottom side of the microchannel has been designed. When an E. coli bacterium comes near the bottom side microelectrodes in the fluid, positive dielectrophoretic force should exerted to trap it near the edge of microelectrodes (between two microelectrodes). As illustrated in Figure 8, in the first part of the microchannel (named as concentrator section) separated targets come near the lower side of the microchannel and as a result in the second part (named as detector section) there is a better chance of trapping them by applying lower voltages. Figure 8 represents the result of solving the particle tracing module of fluid flow by time dependent analysis of COMSOL Multiphysics.
As mentioned earlier, another concerned issue was assuming moving target bacteria as a point that caused differences between results of experimental test and simulation of 2D model. As shown in Figure 9, four different entrance points have been assigned with a distance of 2 $\mu \mathrm{m}$ between them which is equal to the diameter of $E$. coli.

Every four E. coli bacteria entered to the microchannel at the same time, each one from one assigned entrance. Twenty E. coli bacteria went into the microchannel and eighteen of them were trapped in the trapping area (between two bottom side microelectrodes) or near it. Trapped E. coli bacteria shown in Figure 10 after releasing $1,2,5,10,15$ and 20 and trapping 18 of them between or near bottom side microelectrodes.

In the last step, a novel technique has proposed that realized the presence of target bacteria near trapping area by measuring electrical impedance changes. Impedance of water is different from impedance of every solid bio-particles and as a result of this reality the presence of $E$. coli near $p$-DEP microelectrodes cause impedance changing Thus, the exact time and exact position of $E$. coli near detecting microelectrodes has been achieved by calculation of impedance (continuously or discretely with short time difference). As illustrated in Figure 11, impedance analyzer added to the 2D model to calculate variation of impedance between detector microelectrodes (or near them). 


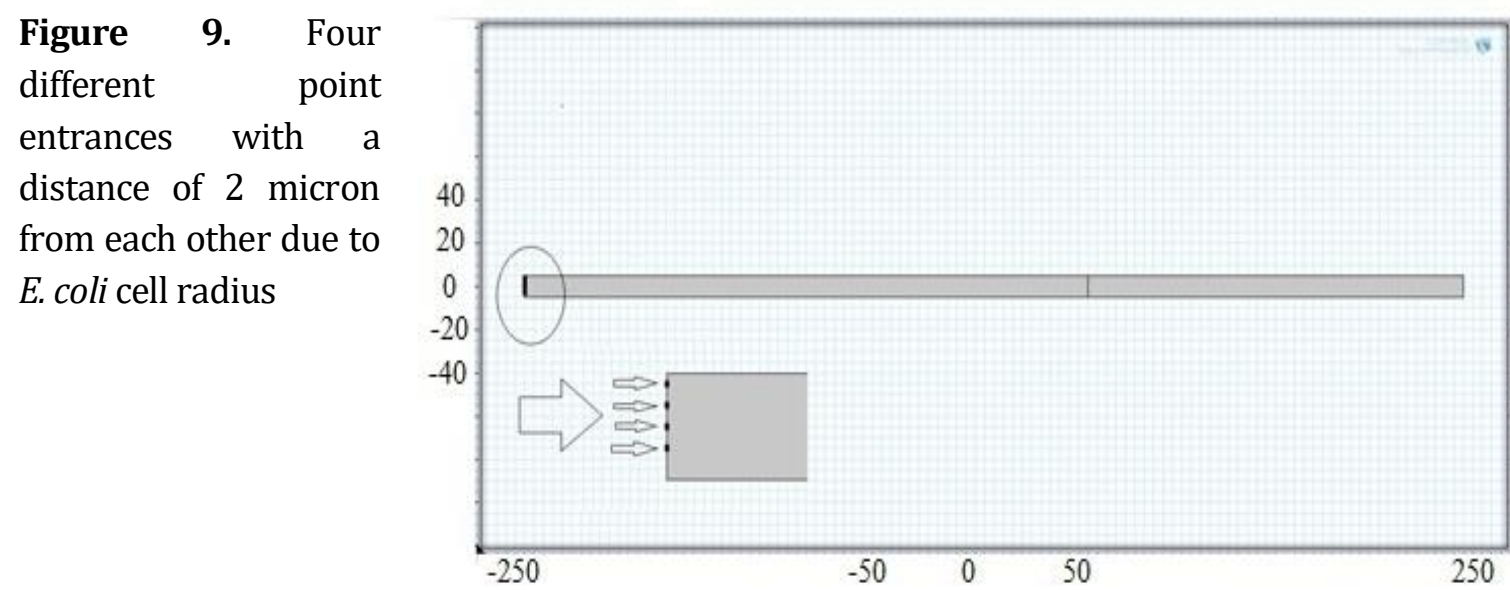

Figure 10. Trapped $E$. coli bacteria after releasing a) 1, b) 2,c) 5 , d) 10 , e) 15 and f) 20 and detecting 18 of them
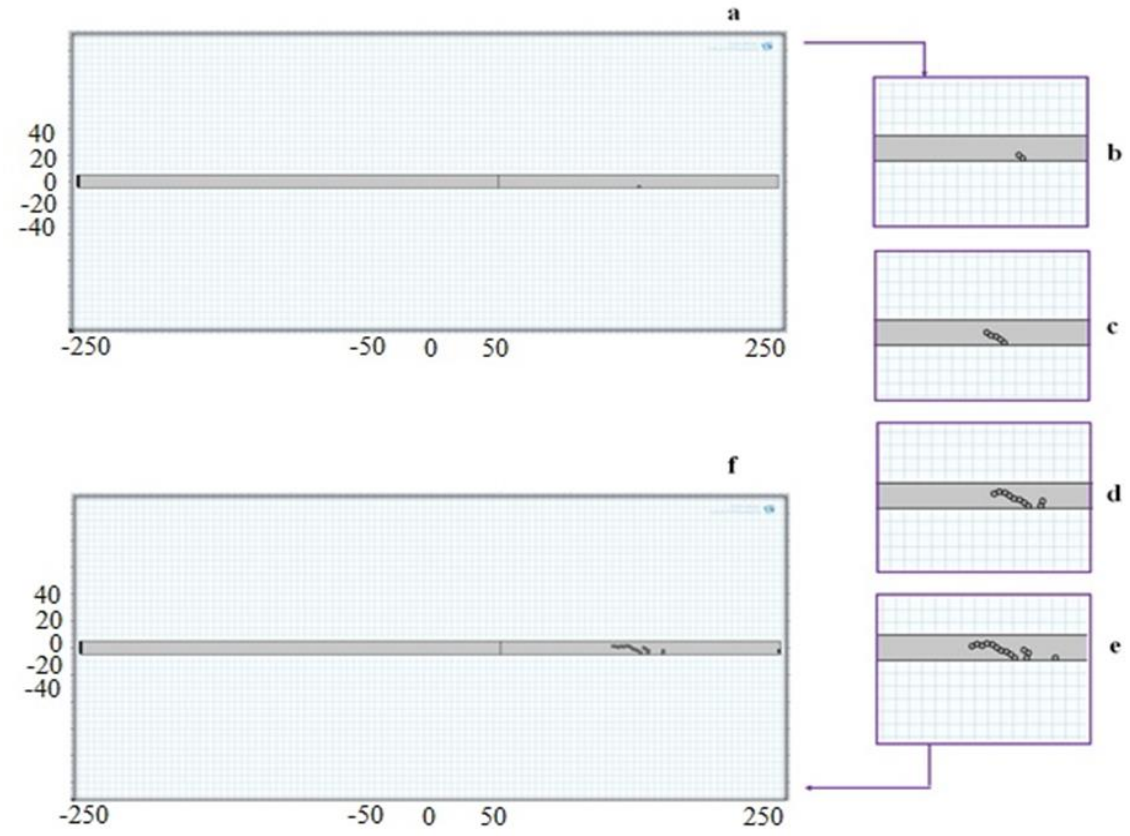

Figure 12 shows impedance versus number of trapped bacteria time dependent analysis of E. coli trajectory and releasing twenty of them in the surrounding medium (water). Thirteenth and seventeenth E. coli were not trapped. They escaped from trapping area and at that time impedance analyzer showed a small change in impedance amount but after that showed the previous amount. It can concluded that they just cross near trapping area. Thus, proposed simple microfluidic system represented better sensitivity than previous models.
The numerical simulation proved that $n$-DEP force could increase DEPIM sensitivity with respect to the absence of the $n$-DEP force. Moreover, as illustrated in Figure 13, this analysis was repeated for one hundred releasing E. coli in different flow rates of the medium. As shown the best flow rate is $27 \mathrm{~cm} / \mathrm{s}$. (based on microchannel dimensions and E. coli simple circular model) and as a result, 80 percent of releasing $E$. coli bacteria trapped near $p$-DEP microelectrodes. 
Figure 11. Concept design of step by step E. coli detection

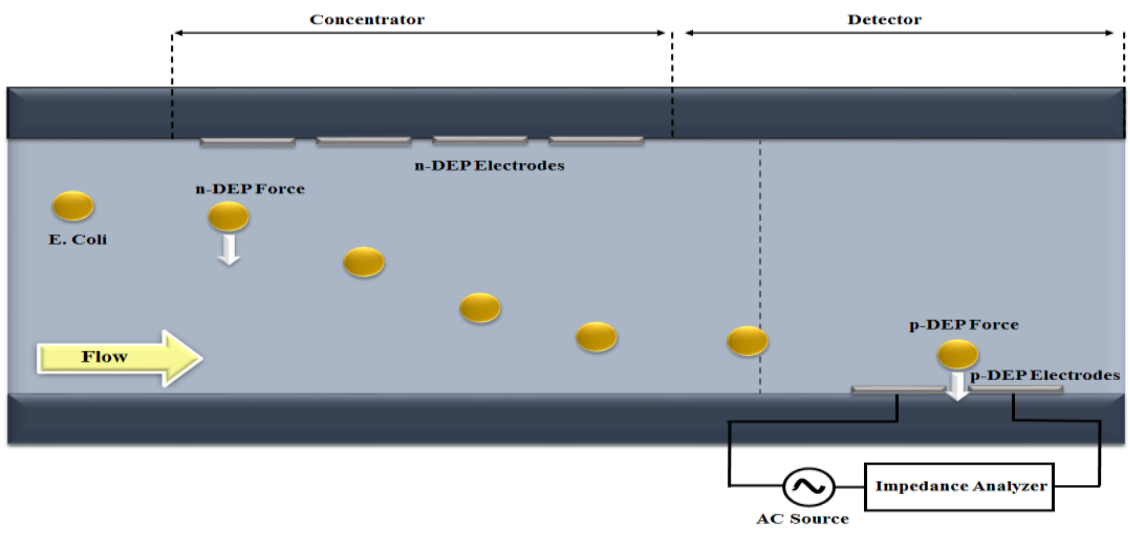

Figure 12. Plot of impedance variation versus number of trapped E. coli bacteria

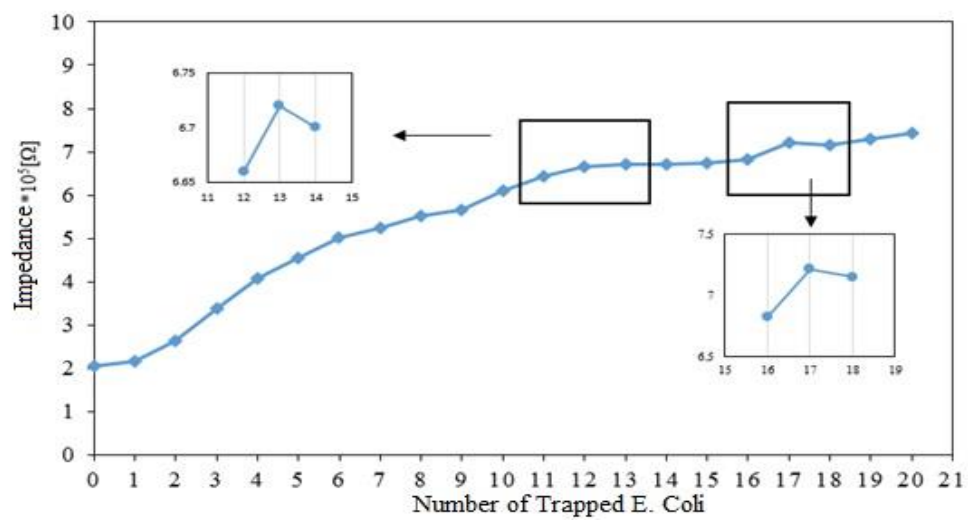

Figure 13. Number of trapped E. coli bacteria versus various flow rate of medium. (One hundred E. coli bacteria have released

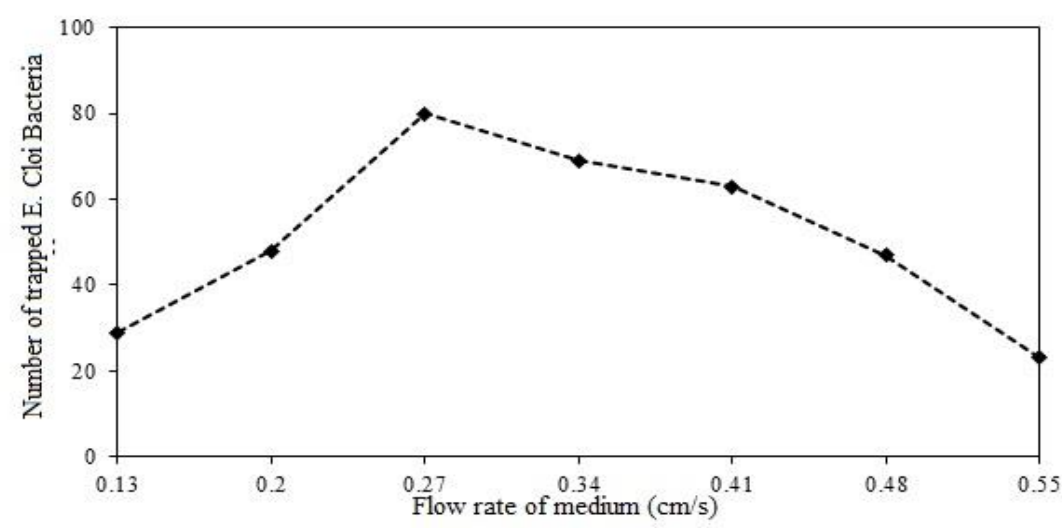

\section{Conclusions}

Conventional DEPIM method is absolutely efficient and reliable bacterial quantitative technique, but the detection period is longer in the case of lower concentration of targets, since it is necessary to trap sufficient amount of the targets to observe huge electrical changes. In this paper, we suggested a 2D model of the $n$ DEP E. coli concentration, which could adapt with DEPIM to improve its sensitivity and efficiency for on-site inspection of E. coli contamination. The simple 2D model has two sections which utilize as an $E$. coli concentrator using $n$-DEP and as an $E$. coli detector using $p$ DEP. Based on theory, effects of the $n$-DEP force as a concentration section have been calculated. In addition, huge number of simulations of different bacteria cell motion have been investigated in the flow microchannel. These calculation results exhibited that suggested $n$ DEP concentrator could influence on the 
trajectory of moving E. coli in the proposed rectangular microchannel and as a result gathered or collected them near the $p$-DEP microelectrodes located on the bottom side of the microchannel. Experimental results of DEPIM, which incorporates $n$-DEP concentrator and $p$-DEP detector sections, showed $n$-DEP concentrator microelectrodes can improve the efficiency of microfluidic systems.

\section{Acknowledgment}

The authors would like to thank the University of Tehran (MEMS and NEMS Laboratory, department of electrical and computer engineering) and Dr. Mohammadreza Kolahdouz.

\section{Disclosure statement}

No potential conflict of interest was reported by the authors.

\section{ORCID}

\section{Fathollahzadeh (D): 0000-0001-9691-6520}

\section{References}

[1] H.A. Pohl, J. Appl. Physics., 1951, 22, 869-871.

[2] I. POHL, Gambridge Monographs on Physics., 1978.

[3] H. Morgan, N.G. Green, Research Studies Press., 2003.

[4] M.P. Hughes, CRC press., 2002.

[5] T.B. Jones, T.B. Jones, Cambridge University Press., 2005.

[6] B.J. Kirby, Cambridge University Press., 2010.

[7] H.C. Chang, L.Y. Yeo, Cambridge University Press, 2010.

[8] R. Hamada, H. Takayama, Y. Shonishi, L. Mao, M. Nakano, J. Suehiro, Sensor. Actuat.. BChem., 2013, 181, 439-445.

[9] J. Voldman, Annu. Rev. Biomed. Eng., 2006, 8, 425-454.

[10] C.F. Gonzalez, V.T. Remcho, J. Chromatography A, 2005, 1079, 59-68.
[11] T.B. Jones, IEEE Eng. Med. Biol. Mag., 2003, $22,33-42$.

[12] L. Linbo, K. Chen, N. Xiang, Z. Ni. Electrophoresis, 2019, 6, 873-889.

[13] C. Siebman, O.D. Velev, V.I. Slaveykova. Biosensors, 2018, 8, 15.

[14] H.J. Kim, D.H. Kang, E. Lee, K. S. Hwang, H.J. Shin, J. Kim, Advanced Biomedical and Clinical Diagnostic and Surgical Guidance Systems XVI International Society for Optics and Photonics., 2018, p 104840I.

[15] R.E. Fernandez, B.J. Sanghavi, V. Farmehini, J.L. Chávez, J. Hagen, N. Kelley-Loughnane, C.F. Chou, N.S. Swami, Electrochem. Commun., 2016, 72, 144-147.

[16] D. Cai, M. Xiao, P. Xu, Y.C. Xu, W. Du, Lab Chip, 2014, 14, 3917-3924.

[17] Y.L. Deng, M.Y. Kuo, Y.J. Juang, Biomicrofluid., 2014, 8, 064120.

[18] M. Fathollahzadeh et al., J. Solid State Electrochem., 2017, 22, 61-67.

[19] L. Fotouhi, M. Fatollahzadeh, M.M. Heravi, Int.J. Electrochem. Sci., 2012, 7, 3919-3928.

[20] M. Fathollahzadeh, M. Hosseini, B. Haghighi, M. Kolahdouz, M. Fathipour, Anal. Chim. Acta, 2016, 924, 99-105.

[21] H. Shayestehpour, K.N. Nazif, A. Soufi, M. Saidi, Scient. Iran. Transact. B, Mechan. Eng., 2018, 25, 186-195.

[22] M. Hosseini, M. Fathollahzadeh, M. Kolahdouz, A. Rostamian, M. Mahmoodian, A. Samaeian, H. H. Radamson, J. Solid State Electrochem., 2018, 22, 3161-3169.

[23] S. Sajed S, K. Vafaei,F. Arefi, M. Fathollahzadeh, M. Kolahdouz M, MA. Sadeghi , M. Neshat, Phys. Status Solidi A, 2019, 1800871.

[24] L. Wang, Small., 2015, 11, 3762-3767.

[25] I. Ermolina, J. Milner, H. Morgan, Electrophoresis, 2006, 27, 3939-3948.

[26] K. Yamada, T.G. Henares, K. Suzuki, D. Citterio, Angew. Chem. Int. Edit., 2015, 54, 5294-5310.

[27] Z. Yang, G. Xu, J. Reboud, S.A. Ali, G. Kaur, J. McGiven, N. Boby, P.K. Gupta, P. Chaudhuri, 
J.M. Cooper., ACS Sensors, 2018, 3, 403409.

[28] A. Alfadhel, J. Ouyang, C.G. Mahajan, F. Forouzandeh, D. Cormier, D.A. Borkholder, Mater. Des., 2018, 150, 182-187.

[29] Z. Bagheri, B. Ranjbar, A. Azizi, H. Latifi, M.I. Zibaii, T. Tohidi Moghadam, Sci. Iran., 2018, 25, 3, 1783-1788.

[30] S. Hiroyuki, Y. Hiruta, D. Citterio, Analyst, 2019, 144, 1178-1186.

[31] N. Gan, L. Xie, K. Zhang, Y. Cao, F. Hu, T. Li, Sensor. Actuat. B-Chem., 2018, 272, 526533.

[32] K. Khoshmanesh, S. Nahavandi, S. Baratchi, A. Mitchell, K. Kalantar zadeh, Bio. Bioelectron., 2011, 26, 1800-1814.

[33] Y. Huang, K.L. Ewalt, M. Tirado, R. Haigis, A. Forster, D. Ackley, M.J. Heller, J.P. O'Connel, M. Krihak ., Anal. chem., 2001, 73, 15491559.

[34] P.R. Gascoyne, J. Vykoukal, Electrophoresis., 2002, 23, 1973-1983.

[35] B.H. Lapizco-Encinas, M. Rito-Palomares, Electrophoresis., 2007, 28, 4521-4538.

[36] J. Suehiro, R. Hamada, D. Noutomi, M. Shutou, M. Hara, J. Electrostatics., 2003, 57, 157-168.

[37] C. Quinn, G. Archer, W. Betts, J. O'Neill, Lett. Appl. Microbiol., 1996, 22, 224-228.

[38] R. Hölzel, J. Electrostatics., 2002, 56, 435447.

[39] D.J. Bakewell, H. Morgan, IEEE Transact. Nanobiosci., 2006, 5, 1-8.

[40] J. Suehiro, T. Hatano, M. Shutou, M. Hara, Sensor. Actuat. B-Chem., 2005, 109, 209215.

[41] M. Nakano, Z. Ding, J. Suehiro, Japan. J. Appl. Phys., 2015, 55, 017001.
[42] J. Chung, Y. Chen, S.J. Kim, Sensor. Actuators B: Chem., 2018, 266, 106-114.

[43] A. Sedaghat, A. Mohamadzadeh, Sci. Iran. Transact. B, Mechan. Eng., 2014, 21, 109118.

[44] W.B. Betts, Trend. Food Sci. Technol., 1995, 6, 51-58.

[45] G.H. Markx, P.A. Dyda, R. Pethig, J. Biotechnol., 1996, 51, 175-180.

[46] R. Pethig, G.H. Markx, Trend. Biotechnol., 1997, 15, 426-432.

[47] W. Betts, A. Brown, J. Appl. Microbiol., 1998, 85, 201-213.

[48] J. Suehiro†, R. Yatsunami, R. Hamada, M. Hara, J. Phys. D: Appl. Phys., 1999, 32, 28142820.

[49] G.H. Markx, Y. Huang, X.F. Zhou, R. Pethig, Microbiology., 1996, 51, 175-180.

[50] N. Manaresi A. Romani, G. Medoro, L. Altomare, A. Leonardi, M. Tartagni, R. Guerrieri, IEEE J. Solid-State Circuits., 2003, 38, 2297-2305.

[51] G. Medoro, N. Manaresi, M. Tartagni, R. Guerrieri, International Electron Devices Meeting, 2000. IEDM'00. Technical Digest. International., 2000, 415-418.

[52] M.P. Hughes, H. Morgan, F.J. Rixon, Biochim. Biophys. Acta (BBA)-General Subjects., 2002, 1571, 1-8.

[53] B. Yafouz, N.A. Kadri, F. Ibrahim, Sensors, 2013, 13, 9029-9046.

[54] G. Falkovich, Cambridge University Press., 2011.

[55] M. Madigan and J. Martinko, Sci. Elo. Espana., 2005.

[56] Z.D. Blount, C.Z. Borland, R.E. Lenski, Proce. Natl. Acad. Sci., 2008, 105, 7899-7906.

[57] M.S. Donnenberg, Academic Press., 2002.

How to cite this manuscript: Alireza Ebrahimi, Morteza Fathipour, Maryam Fathollahzadeh, An Improved Escherichia Coli Bacterium Detection in icrochannel Based on Dielectrophoresis Impedance Measurements, Adv. J. Chem. A, 2020, 3(2), 131-145. 